\title{
Цепочечная модель декорирования зигзагообразной кромки графена
}

\author{
(C) С.Ю. Давыдов \\ Физико-технический институт им. А.Ф. Иоффре Российской академии наук, \\ 194021 Санкт-Петербург, Россия \\ E-mail: Sergei_Davydov@mail.ru
}

(Получена 18 июня 2018 г. Принята к печати 6 июля 2018 г.)

На решетке типа „кирпичной стенки“, топологически эквивалентной решетке графена, построена простая структурная модель зигзагообразной кромки, декорированной частицами. Получены аналитические выражения для зонного спектра, плотностей состояний и чисел заполнения для системы графен-частицы, находящейся как в свободном состоянии, так и сформированной на металлической подложке.

DOI: 10.21883/FTP.2019.01.46992.8939

\section{1. Введение}

Декорирование островков графена (чешуек, хлопьев, или flakes) путем присоединения к краям чужеродных атомов или молекул продиктовано желанием управлять характеристиками таких квантовых точек [1-3]. В соответствующих теоретических работах рассматриваются графеновые наноленты [1,2], квадратные и треугольные островки [3]. С теоретической точки зрения сходные задачи возникали ранее при изучении кромок графита $[4,5]$ и контактов латеральных графеноподобных структур [6-10]. Во всех цитированных работах отмечается, что особый интерес представляют зигзагообразные края, электронный спектр которых характеризуется пиком плотности состояний вблизи точки Дирака. К недостаткам этих работ, однако, следует отнести то обстоятельство, что в них рассматривались только свободные графеновые листы, хотя условием их существования в приборных структурах является наличие подложки.

В настоящей работе мы рассмотрим как свободный, так и эпитаксиальный графен (эпиграфен) и взаимодействующую с его зигзагообразной кромкой адсорбированную частицу (адчастицу). При этом для описания эпиграфена применяется адсорбционная модель [11,12], а для определения электронной структуры его зигзагообразной кромки используется простейшая, по-видимому, цепочечная модель. В качестве подложки будем рассматривать металл. Отметим, что проблема декорирования кромки эпиграфена сводится к задаче о взаимодействии чужеродных частиц как с кромкой эпиграфена, так и непосредственно с металлической подложкой.

\section{2. Модель: общие соотношения и зонная структура}

Воспользуемся для описания двумерной гексагональной структуры графена топологически эквивалентной решеткой типа кирпичной стенки $[13,14]$ с расстоянием $a$ между тремя ближайшими соседями (б.с.).
На рис. 1 изображена структурная модель декорирования кромки эпиграфена. Отметим, что модель соответствует именно зигзагообразной кромке, так как адчастицы (адсорбированные атомы, молекулы, радикалы) связаны с каждым вторым краевым адатомом графена. Изолированным адатомам углерода и адчастице припишем соответственно затравочные функции Грина

$$
g=(\omega+i \Gamma)^{-1}, \quad g_{a}=\left(\omega-\varepsilon_{a}+i \Gamma_{a}\right)^{-1},
$$

где энергия $p$-состояния адатома углерода принята за нуль, $\varepsilon_{a}$ - энергия уровня адчастицы, $Г$ и $\Gamma_{a}-$ затухания электронных состояний (полуширина квазиуровней) адатомов углерода и адчастицы [11]. Воспользовавшись уравнением Дайсона [11] и нумерацией адатомов $(x, y)$, представленной на рис. 1, и считая, что энергия перехода между б. с. в графене равна $t$, а между адатомами графена и адчастицами равна $\tilde{t}$, запишем цепочку уравнений

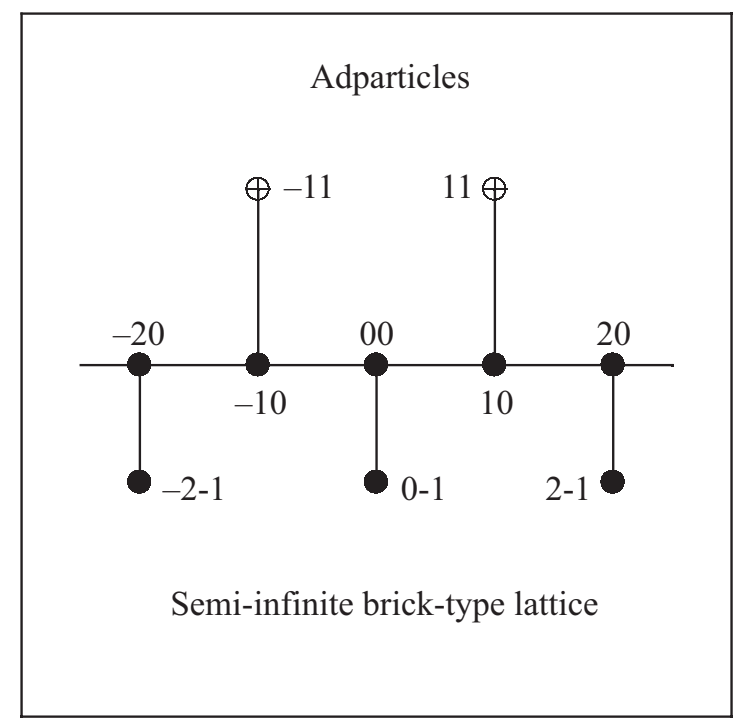

Рис. 1. Структурная модель декорированной зигзагообразной кромки графена. Адатомы углерода обозначены темными кружками, адчастицы - светлыми перекрещенными кружками. 
вида

$$
\begin{gathered}
G_{00,00}=g+g t G_{0-1,00}+g t\left(G_{-10,00}+G_{10,00}\right), \\
G_{0-1,00}=g t G_{00,00}, \\
G_{-10,00}=g t\left(G_{-20,00}+G_{00,00}\right)+g \tilde{t} G_{-11.00}, \\
G_{10,00}=g t\left(G_{20,00}+G_{00,00}+g \tilde{t} G_{11,00}\right), \\
G_{-11,00}=g_{a} \tilde{t} G_{-10,00}, \quad G_{11,00}=g_{a} \tilde{t} G_{10,00} .
\end{gathered}
$$

Тогда для адатома графена, находящегося в узле (00) (рис. 1), получим функцию Грина вида

$$
G_{00,00} \equiv G_{1}(\omega, k)=g\left(1-g^{2} t^{2}-\frac{4 g^{2} t^{2} \cos ^{2}(k a)}{1-g g_{a} \tilde{t}^{2}}\right)^{-1},
$$

где $k=k_{x},|k| \leq \pi / 2 a$. Аналогичным образом для адатома графена в узле (10) найдем

$g_{10,10} \equiv G_{2}(\omega, k)=g\left(1-g g_{a} \tilde{t}^{2}-\frac{4 g^{2} t^{2} \cos ^{2}(k a)}{1-g^{2} t^{2}}\right)^{-1}$.

Определим теперь функцию Грина адчастицы, занимающей узел (11). Исходя из уравнения Дайсона получим следующую цепочку уравнений:

$$
\begin{gathered}
G_{11,11}=g_{a} \tilde{t} G_{10,11}, \\
G_{10,11}=\operatorname{gt}\left(G_{20,11}+G_{0,11}\right)+g \tilde{t} G_{11,11}, \\
G_{20,11}=\operatorname{gt}\left(G_{30,11}+G_{10,11}+G_{2-1,1^{\prime} 1}\right), \\
G_{00,11}=\operatorname{gt}\left(G_{-10,11}+G_{10,11}+G_{0-1,1^{\prime} 1}\right),
\end{gathered}
$$

откуда следует, что

$$
G_{11,11} \equiv G_{a}(\omega, k)=g_{a}\left(1-\frac{g g_{a} \tilde{t}^{2}}{1-\frac{4 g^{2} t^{2} \cos ^{2}(k a)}{1-g^{2} t^{2}}}\right)^{-1} .
$$

Аналогичным образом найдем

$$
G_{0-1,0-1} \equiv G_{-1}(\omega, k)=g\left(1-\frac{g^{2} t^{2}}{1-\frac{4 g^{2} t^{2} \cos ^{2}(k a)}{1-g g_{a} \tilde{t}^{2}}}\right)^{-1} .
$$

Полюса функций Грина (2)-(5), отвечающие уравнению

$$
\begin{aligned}
A(\omega, k) & \equiv\left(\omega^{2}-t^{2}\right)\left[\omega\left(\omega-\varepsilon_{a}-\tilde{t}^{2}\right)\right]-\omega\left(\omega-\varepsilon_{a}\right) t^{2} \Phi(k) \\
& =0
\end{aligned}
$$

где $\Phi(k)=4 \cos ^{2}(k a)$, определяют зонную структуру $\omega(k)$ рассматриваемой системы. На границе зоны Бриллюэна при $k a=\pi / 2$ имеем решения

$$
\omega= \pm t, \quad \omega=\left[\varepsilon_{a} \pm \sqrt{\varepsilon_{a}^{2}+4 \tilde{t}^{2}}\right] / 2 .
$$

Отметим, что уравнение (6) инвариантно относительно одновременной замены знаков $\varepsilon_{a}$ и $\omega$.

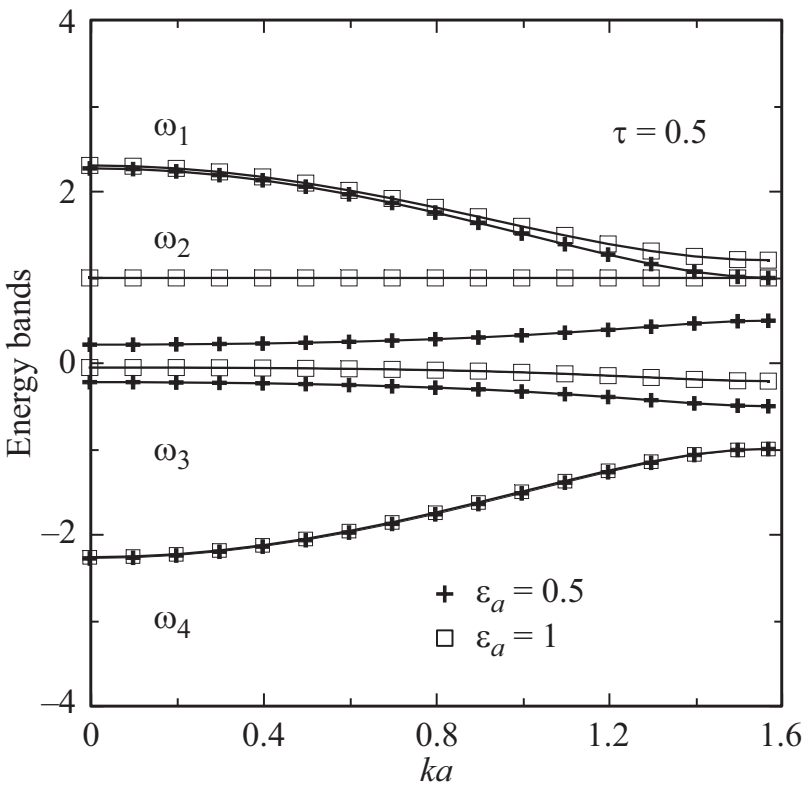

Рис. 2. Энергетические зоны $\omega_{i}(k)$ при $\tau=0.5$ и $\varepsilon_{a}=t$ (квадраты), $\varepsilon_{a}=0.5 t$ (кресты). Все энергетические величины на рисунке приведены в ед. $t$.

Дисперсионные зависимости $\omega_{i}(k)$ представлены на рис. 2, откуда в первую очередь следует, что наличие адчастиц существенно сказывается только на низкоэнергетических зонах $\omega_{2}(k)$ и $\omega_{3}(k)$, а удаленные от точки Дирака зоны $\omega_{1}(k)$ и $\omega_{4}(k)$ почти не изменяются. При $\varepsilon_{a}=0$ из уравнения (6) получаем четыре зоны вида

$$
\begin{aligned}
\omega(k)= & \pm(t / \sqrt{2})\left(1+\tau^{2}+\Phi(k)\right. \\
& \left. \pm \sqrt{\left(1-\tau^{2}\right)^{2}+2\left(1+\tau^{2}\right) \Phi(k)+\Phi^{2}(k)}\right)^{1 / 2}
\end{aligned}
$$

где $\tau=\tilde{t} / t$. Эти зоны симметричны (рис. 2) относительно нулевой энергии: $\omega_{4}(k)=-\omega_{1}(k)$ и $\omega_{3}(k)=-\omega_{2}(k)$. Если же $\varepsilon_{a} \neq 0$, то симметрия нарушается. Отметим, что случай $\varepsilon_{a}=0, \tau=1$ отвечает модели узкой графеновой наноленты [15], с зонами

$$
\omega(k)= \pm t\left(1+2 \cos ^{2}(k a) \pm 2 \cos (k a) \sqrt{1+\cos ^{2}(k a)}\right)^{1 / 2} .
$$

\section{3. Декорирование свободного графена}

\section{1. Плотности состояний}

Анализ плотностей состояний начнем со случая свободных (не связанных с подложкой) графена и цепочки частиц, взаимодействующих с его краем, положив $\Gamma=\Gamma_{a}=s=0^{+}$и приписав функциям Грина верхний индекс 0. Для дальнейшего функции Грина (2) и (3) удобно представить в виде $G_{1,2}^{0}(\omega, k)=C_{1,2}(\omega) / A(\omega, k)$, 
где $C_{1}(\omega)=\omega\left[\omega\left(\omega-\varepsilon_{a}\right)-\tilde{t}^{2}\right], C_{2}(\omega)=\left(\omega-\varepsilon_{a}\right)\left(\omega^{2}-t^{2}\right)$. Тогда, интегрируя по зоне Бриллюэна, получим

$$
G_{1,2}^{0}(\omega)=\frac{a}{\pi} \int_{-\pi / 2 a}^{\pi / 2 a} G_{1,2}^{0}(\omega, k) d k=\frac{C_{1,2}(\omega)}{D \sqrt{1-B^{2}}},
$$

где $B=2 \omega\left(\omega-\varepsilon_{a}\right) t^{2} / D, D=\left(\omega^{2}-t^{2}\right)\left[\omega\left(\omega-\varepsilon_{a}\right)-\tilde{t}^{2}\right]$ $-2 \omega\left(\omega-\varepsilon_{a}\right) t^{2}$. Так как $\rho_{1,2}^{0}(\omega)=-\pi^{-1} \operatorname{Im} G_{1,2}^{0}(\omega)$ и
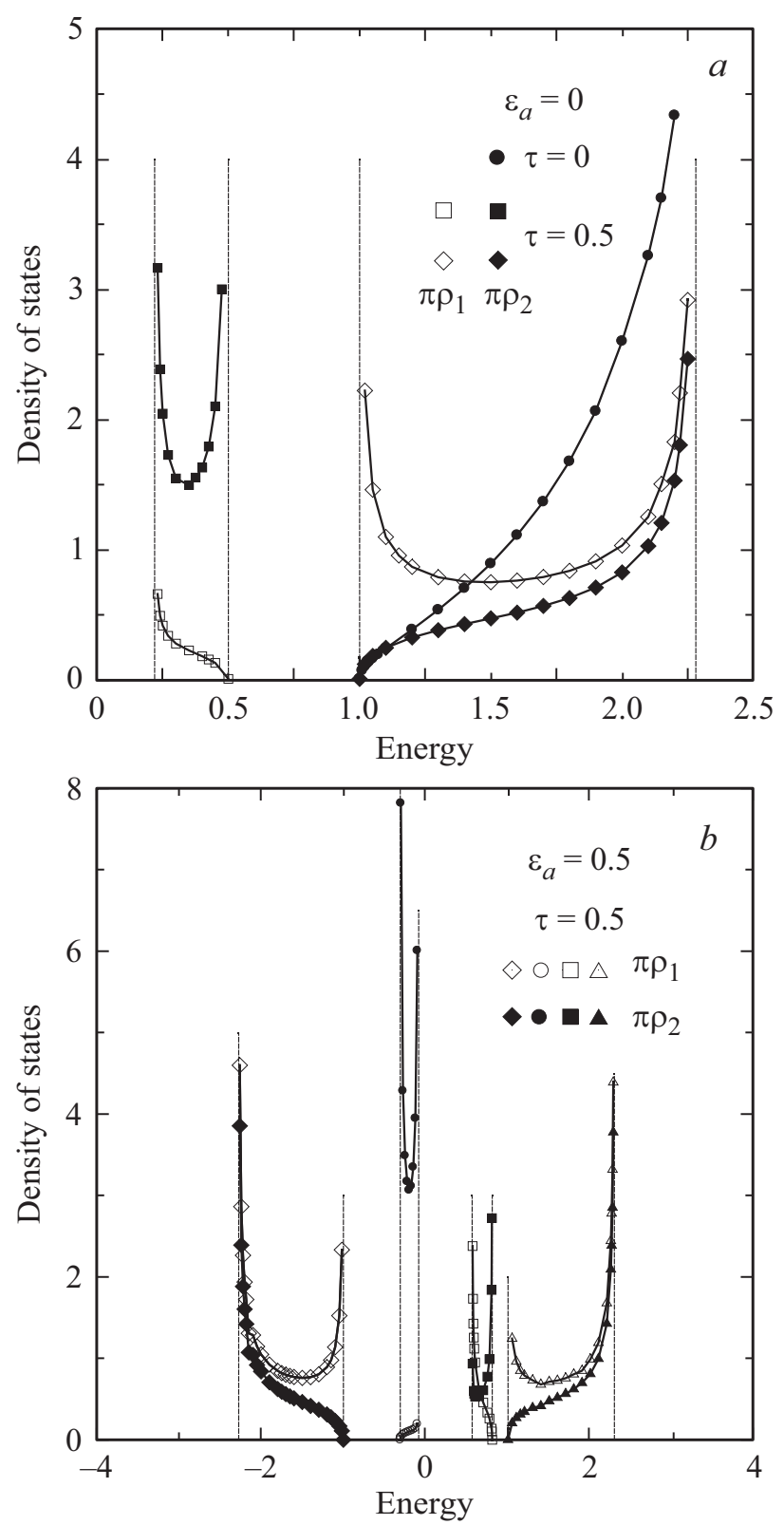

Рис. 3. Плотности состояний $\rho_{1}^{0}(\omega)$ и $\rho_{2}^{0}(\omega)$ (светлые и темные символы соответственно) в отсутствие подложки для различных значений параметров задачи $\varepsilon_{a}$ и $\tau$. В случае $\varepsilon_{a}=0$ и $\tau=1$ (темные кружки) имеем $\rho_{1}^{0}(\omega)=\rho_{2}^{0}(\omega)$. Все энергетические величины измеряются в ед. $t$, плотности состояний в ед. $t^{-1}$. На рис. $3, a$ изображены только правые половины функций $\rho_{1}^{0}(\omega)$ и $\rho_{2}^{0}(\omega)$, симметричных относительно $\omega=0$. Разрывы функций $\rho_{1}^{0}(\omega)$ и $\rho_{2}^{0}(\omega)$ на границах зон обозначены пунктирными асимптотами. зонные плотности состояний отвечают случаю $B^{2} \geq 1$, имеем

$$
\rho_{1,2}^{0}(\omega)=\frac{1}{\pi} \frac{\left|C_{1,2}(\omega) / D\right|}{\sqrt{B^{2}-1}} .
$$

Аналогичным образом представим функции Грина (4) и (5) в виде $G_{a,-1}^{0}(\omega, k)=C_{a,-1}(\omega, k) / A(\omega, k)$, где $C_{a}(\omega, k)=\omega\left[\omega^{2}-t^{2}(1+\Phi(k))\right], C_{-1}(\omega, k)=\omega\left[\omega\left(\omega-\varepsilon_{a}\right)\right.$ $\left.-\tilde{t}^{2}\right]-\left(\omega-\varepsilon_{a}\right) t^{2} \Phi(k)$. Перепишем $C_{a,-1}(\omega, k)$ в виде $C_{a,-1}(\omega, k)=C_{a,-1}^{(1)}(\omega)+C_{a,-1}^{(2)}(\omega) \cos (2 k a)$, где $C_{a}^{(1)} \omega=$ $=\omega\left(\omega^{2}-3 t^{2}\right), C_{a}^{(2)}(\omega)=-2 \omega t^{2}, C_{-1}^{(1)}(\omega)=\omega\left[\omega\left(\omega-\varepsilon_{a}\right)\right.$ $\left.-\tilde{t}^{2}\right]-2\left(\omega-\varepsilon_{a}\right) t^{2}, C_{-1}^{(2)}=-2\left(\omega-\varepsilon_{a}\right)^{2}$. Интегрируя по зоне Бриллюэна, получим

$$
\begin{aligned}
& G_{a,-1}^{0}(\omega)=\frac{2 a}{\pi} \int_{0}^{\pi / 2 a} G_{a,-1}^{0}(\omega, k) d k \\
& =\frac{1}{D \sqrt{1-B^{2}}}\left(C_{a,-1}^{(1)}(\omega)+C_{a,-1}^{(2)}(\omega) \frac{\sqrt{1-B^{2}}-1}{|B|}\right),
\end{aligned}
$$

так что при $B^{2} \geq 1$ и $\rho_{a,-1}^{0}(\omega)=-\pi^{-1} \operatorname{im} G_{a,-1}^{0}(\omega)$ имеем

$$
\rho_{a,-1}^{0}(\omega)=\frac{1}{\pi} \frac{\left|C_{a,-1}(\omega) / D\right|}{\sqrt{B^{2}-1}},
$$

где $C_{a,-1}(\omega)=C_{a,-1}^{(1)}(\omega)-C_{a,-1}^{(2)}(\omega) /|B|$. На рис. 3 показаны как частные случаи $(a)$, так и общий случай $(b)$ плотностей состояний $\rho_{1,2}^{0}(\omega)$. Отметим два типа зависимостей $\rho_{1,2}^{0}(\omega)$. К первому типу относятся плотности состояний, испытывающие разрывы на обеих границах зон. Такие плотности состояний характерны для одноатомных цепочек [16] (см. также модель Лаббе-Фриделя [17]). Ко второму типу относятся плотности состояний, имеющие разрыв только на одной границе, так как расходимость на второй границе компенсируется обнулением множителя $C_{1,2}(\omega)$. Из сравнения выражений (10) и (12) следует, что с качественной точки зрения плотности состояний $\rho_{1,2}^{0}(\omega)$ и $\rho_{a,-1}^{0}(\omega)$ эквивалентны, отличны лишь $C$-коэффициенты. Поэтому здесь мы ограничились только графиками $\rho_{a, 2}^{0}(\omega)$. Отметим, что плотность состояний при $\varepsilon_{a}=0$ и $\tau=1$ (рис. $3, a$, темные кружки) соответствует наноленте графена.

\section{2. Числа заполнения}

Перейдем теперь к оценкам чисел заполнения. Из общих соображений ясно, что в случае $\varepsilon_{a}=0$ переход заряда между адчастицей и графеном отсутствует. Поэтому с точки зрения допирования графена путем декорирования интерес представляют только случаи $\varepsilon_{a} \neq 0$. В принципе здесь также можно рассмотреть предел $\left|\varepsilon_{a}\right| \ll \tilde{t} \leq t$. Однако даже в этом пределе не удастся получить простые аналитические результаты. Поэтому прибегнем к упрощению, учитывая, что наибольший вклад в $n_{i}^{0}$, где $i=1,2, a,-1$, дают области расходимостей функций $\rho_{i}^{0}(\omega)$, соответствующие границам зон. 
Рассмотрим интеграл

$$
\begin{aligned}
I_{n}(\omega) & =\int_{\omega_{n b}}^{\omega} \frac{d \omega}{\sqrt{\left(\omega_{n t}-\omega\right)\left(\omega-\omega_{n b}\right)}} \\
& =2 \arctan \sqrt{\frac{\omega-\omega_{n b}}{\omega_{n t}-\omega}},
\end{aligned}
$$

где $\omega_{n b}-$ нижняя граница $n$-зоны $(n=1,2,3,4$, см. рис. $2, b)$. Отметим, что $I_{i}\left(\omega_{n t}\right)=\pi$, где $\omega_{n t}-$ верхняя граница $n$-зоны. Тогда, интегрируя плотности состояний (10) и (12) по энергии от дна нижней зоны до уровня Ферми $\varepsilon_{\mathrm{F}}$, получим для чисел заполнения $i$-го атома $n_{i}^{0}$ выражение

$$
n_{i}^{0}=2 \pi^{-1} \sum_{n} \nu_{i n} I_{n}\left(\Sigma_{\mathrm{F}}\right) \Theta\left(\varepsilon_{\mathrm{F}}-\omega_{n b}\right) .
$$

Здесь $\Theta(.$.$) - функция Хэвисайда, а весовые множите-$ ли $v_{i n}$, отвечающие вкладу $n$-й зоны в число заполнения $i$-го атома, равны

$$
\begin{gathered}
v_{i n}=\frac{1}{2}\left(\frac{\left|C_{t}\left(\omega_{n b}\right)\right|}{\sqrt{\left|\prod_{\alpha^{\prime} \neq n b}\left(\omega_{n b}-\omega_{\alpha^{\prime}}+i s\right)\right|}}\right. \\
\left.+\frac{\left|C_{i}\left(\omega_{n t}\right)\right|}{\sqrt{\left|\prod_{\alpha^{\prime} \neq n t}\left(\omega_{n t}-\omega_{\alpha^{\prime}}+i s\right)\right|}}\right),
\end{gathered}
$$

где энергии границ зон $\omega_{\alpha}$ являются корнями уравнения $B= \pm 1$, или уравнения $A(\omega, 0)=0$. Отметим, что $C_{-1}\left(\omega_{\alpha}\right)=C_{1}\left(\omega_{\alpha}\right)$, так как $\left|B\left(\omega_{\alpha}\right)\right|=1$. Считая, что в отсутствие подложки в системе заполнены только зоны с отрицательной энергией, получим для случая $\varepsilon_{a}=0.5 t$, $\tau=0.5$ следующие числа заполнения: $n_{1}^{0}=n_{-1}^{0} \approx 0.79$, $n_{2}^{0} \approx 1.74, n_{a}^{0} \approx 0.68$. При этом мы немного скоррелировали результаты расчетов по формулам (14), (15), учтя (исходя из закона сохранения заряда), что должно выполняться тождество $\sum_{\alpha} v_{i \alpha}=1$. Отметим, что аналогичный метод оценки для чисел заполнения использовался в работах $[10,18]$, где, однако, это тождество выполнялось автоматически. Дело в том, что в $[10,18]$ в знаменатели выражений вида (15) входят полюса 1-го порядка, а не корневые особенности, как в настоящей работе.

\section{4. Декорирование эпиграфена}

Для выяснения влияния металлической подложки прибегнем к дальнейшим упрощениям и рассмотрим квазисвободный (т.е. слабо связанный с субстратом) эпиграфен, полагая, что $\Gamma \ll t$. Предположим, что в свободном состоянии плотность состояний $\rho_{i}^{0}(\omega)$ с достаточной точностью может быть описана выражением

$$
\rho_{i}^{0}(\omega)=\pi^{-1} \sum_{n} v_{i n}\left[\delta\left(\omega-\omega_{n b}\right)+\delta\left(\omega-\omega_{n t}\right)\right] .
$$

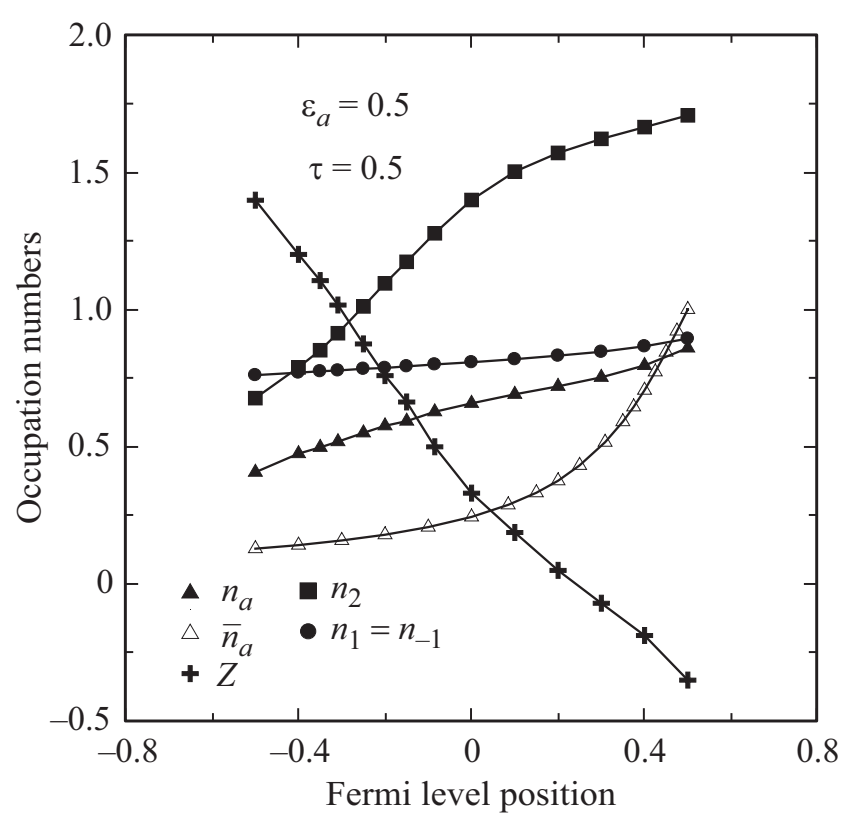

Рис. 4. Зависимости чисел заполнения адатомов углерода $\left(n_{1}=n_{-1}, n_{2}\right)$, адчастицы, связанныой $\left(n_{a}\right)$ и несвязанной $\left(\bar{n}_{a}\right)$ с графеном, и перехода заряда $Z=4-\sum_{i} n_{i}$ между адслоем и металлической подложкой от положения уровня Ферми $\varepsilon_{\mathrm{F}}$.

Легко показать, что сумма в выражении (16) тождественна $\quad \sum_{\alpha} v_{i \alpha} \delta\left(\omega-\omega_{\alpha}\right), \quad$ где $\quad v_{i \alpha}=$ $=\left|C_{i}\left(\omega_{\alpha}\right)\right| / 2 \sqrt{\prod_{\alpha^{\prime} \neq \alpha}\left(\omega_{\alpha}-\omega_{\alpha}+i s\right)}$. При наличии металлической подложки, аналогично $[10,18]$, запишем

$$
\rho_{i}(\omega)=\sum_{\alpha} v_{i \alpha} \rho^{\alpha}(\omega), \quad n_{i}=2 \sum_{\alpha} v_{i \alpha} n^{\alpha},
$$

где $\quad \rho^{\alpha}(\omega)=\Gamma / \pi\left[\left(\omega-\omega_{\alpha}\right)^{2}+\Gamma^{2}\right] \quad$ и $\quad n^{\alpha}=$ $=\pi^{-1} \operatorname{arcctg}\left[\left(\omega_{\alpha}-\varepsilon_{\mathrm{F}}\right) / \Gamma\right]$. Таким образом, в рамках предлагаемой схемы, в присутствии металлической подложки плотность состояний системы $\rho_{i}(\omega)$ представляет собой суперпозицию распределений Лоренца. С ростом Г зависимость $\rho_{i}(\omega)$ сглаживается: высота пиков уменышается, а их ширина растет. При смещении уровня Ферми вверх по шкале энергии числа заполнения $n_{i}$ монотонно увеличиваются, а характерные для свободного графены ступенчатые зависимости $n_{i}\left(\varepsilon_{\mathrm{F}}\right)$ вида (14) сглаживаются.

На рис. 4 для случая $\varepsilon_{a}=0.5, \tau=0.5$ представлены зависимости $n_{i}\left(\varepsilon_{\mathrm{F}}\right)$, а также переход заряда $Z\left(\varepsilon_{\mathrm{F}}\right)=4-\sum_{i} n_{i}\left(\varepsilon_{\mathrm{F}}\right)$. Там же для сравнения представлен график $\bar{n}_{a}\left(\varepsilon_{\mathrm{F}}\right)=(2 / \pi) \operatorname{arccot}\left[\left(\varepsilon_{a}-\varepsilon_{\mathrm{F}}\right) / \Gamma\right]$ для изолированной (не связанной с кромкой эпиграфена) адчастицы. Так как работа выхода графена $\phi_{\mathrm{Gr}} \sim 4.5$ эB и $t \sim 3$ эВ, рассмотренный нами интервал работ выхода $\phi_{\mathrm{M}}=3-6$ эВ перекрывает по сути весь возможный набор металлических подложек (см. значения $\phi_{\mathrm{M}}$ для граней (111) в [19]). Как следует из рис. 4, с ростом $\varepsilon_{\mathrm{F}}$ все числа заполнения увеличиваются, а 
переход заряда уменьшается. В наибольшей степени растет значение $n_{2}\left(\varepsilon_{\mathrm{F}}\right)$, тогда как $n_{1}\left(\varepsilon_{\mathrm{F}}\right)=n_{-1}\left(\varepsilon_{\mathrm{F}}\right)$ почти не изменяется. Отметим также, что значения чисел заполнения $\bar{n}_{a}$ и $n_{a}$ и их зависимости от $\varepsilon_{\mathrm{F}}$ значительно различаются.

При $\varepsilon_{\mathrm{F}}=0$ получаем $n_{1}=n_{-1} \approx 0.81, n_{2} \approx 1.40$, $n_{a} \approx 0.66, \quad Z \approx 0.32$. Сравнение с рассчитанными в предыдущем разделе значениями $n_{a}^{0}=n_{-1}^{0} \approx 0.79$, $n_{2}^{0} \approx 1.74, n_{a}^{0} \approx 0.68$ показывает, что отток электронов в подложку идет с адатомов углерода типа $(-20),(00)$ и (02) (рис. 1). Подчеркнем, что случай $\varepsilon_{\mathrm{F}}=0$ отнюдь не является чисто академическим, так как, например, для $\mathrm{Cu}(111)$ и $\mathrm{Mo}(111)$ работа выхода равна 4.55 эВ, а для $\mathrm{W}(111)-4.47$ эB [19], что почти совпадает с $\phi_{\mathrm{Gr}}$ (стоит, однако, иметь в виду, что данные по $\phi_{\mathrm{Gr}}$ и $\phi_{\mathrm{M}}$ имеют заметный разброс - см., например, $[19,20])$. Напомним [11], что для бесконечного листа эпиграфена при $\phi_{\mathrm{Gr}}=\phi_{\mathrm{M}}$ переход заряда между адатомами углерода и металлом отсутствует.

Поясним теперь выбор интервала значений $\varepsilon_{a}=3$ эВ, что соответствует уровню частицы, лежащему на 4.5-1.5 эВ ниже уровня вакуума. Согласно теории адсорбции [11], можно положить $\varepsilon_{a}=\phi_{\mathrm{Gr}}-(I+A) / 2$ [21], где энергия уровня отсчитывается от точки Дирака, $I$ и $A$ - потенциал ионизации и энергия сродства к электрону декорирующей частицы. Этому энергетическому интервалу соответствуют металлические атомы [19]. Уровням атомов и молекул газа отвечает интервал $\varepsilon_{a}=(-t)-0$, т. е. на $7.5-4.5$ эВ ниже уровня вакуума. В связи с этим напомним, что определяющее закон дисперсии уравнение (6) инвариантно относительно замены $\varepsilon_{a}$ на $-\varepsilon_{a}$ и $\omega$ на $-\omega$. Ту же инвариантность демонстрируют плотности состояний (10), (12). Отсюда следует, что $n_{i}^{0}\left(\varepsilon_{a}\right)=2-n_{i}^{0}\left(-\varepsilon_{a}\right)$. Следовательно, при $\varepsilon_{a}=-0.5 t, \quad \tau=0.5, \quad \varepsilon_{\mathrm{F}}=0$ числа заполнения равны $n_{1}^{0}=n_{-1}^{0} \approx 1.21, n_{2}^{0} \approx 0.26$, $n_{a}^{0} \approx 1.32$. В присутствии подложки справедливы аналогичные соотношения: $\rho^{\alpha}\left(\omega, \omega_{\alpha}\right)=\rho^{\alpha}\left(-\omega,-\omega_{\alpha}\right)$ и $n^{\alpha}\left(\omega_{\alpha}, \varepsilon_{\mathrm{F}}\right)=2-n^{\alpha}\left(-\omega_{\alpha},-\varepsilon_{\mathrm{F}}\right) \quad$ (см. (17)), так что $\quad n_{i}\left(\varepsilon_{a}, \varepsilon_{\mathrm{F}}\right)=2-n_{i}\left(-\varepsilon_{a},-\varepsilon_{\mathrm{F}}\right), \quad Z\left(\varepsilon_{a},-\varepsilon_{\mathrm{F}}\right)=$ $=-Z\left(-\varepsilon_{a},-\varepsilon_{\mathrm{F}}\right)$. Поэтому для случая $\varepsilon_{a}=-0.5 t$, $\tau=0.5$ можно использовать рис. 4 , поменяв знак $\varepsilon_{F}$ и отсчитывая $n_{i}$ от 2 . В частности, при $\varepsilon_{\mathrm{F}}=0$ имеем $n_{1}=n_{-1} \approx 1.19, n_{2} \approx 0.60, n_{a} \approx 1.34, Z \approx-0.32$.

Параметр $\Gamma=\pi \rho_{\mathrm{M}} V^{2}$, где $\rho_{\mathrm{M}}$ - плотность состояний металлической подложки, $V$ - матричный элемент связи графена и адчастицы с металлическим субстратом $[11,16]$. В адсорбционной связи участвуют ковалентное и ван-дер-ваальсово взаимодействия. Соответствующие схемы оценок Г приведены в [22]. И наконец, полагая в соответствии с методом связывающих орбиталей Харрисона [23], что матричные элементы $t \propto a^{-2}, \tilde{t} \propto \tilde{a}^{-2}$, где $\tilde{a}-$ длина связи частицы с углеродом кромки графена, в случае $\tau=0.5$ получим $\tilde{a}=a \sqrt{2} \approx 2 \AA$ (для графена $a=1.42 \AA$ ), что вполне разумно [22].

\section{5. Обсуждение результатов и заключительные замечания}

Цель модельного подхода к проблеме заключается в определении зависимостей тех или иных характеристик от параметров задачи. Поэтому, как правило, прямое сравнение полученных в рамках простых моделей результатов с результатами расчетов для той или иной конкретной системы затруднительно. Попытаемся, однако, провести хотя бы качественное сравнение.

В работе [1] рассматривалась графеновая нанолента, одна из зигзагообразных кромок которой декорирована водородом, а другая - кетоном, карбонильными, карбоксильными и гидроксильными группами. Показано, что работа выхода края, содержащего гидроксильные группы (связь $\mathrm{C}(\mathrm{Gr})-\mathrm{OH}$, где $\mathrm{C}(\mathrm{Gr})$ - атом углерода графена), ниже работы выхода края, содержащего водород, в то время как для края с кетоном $(\mathrm{C}(\mathrm{Gr})-\mathrm{O})$, карбонильной $(\mathrm{C}(\mathrm{Gr})-\mathrm{COH})$ и карбоксильной $(\mathrm{C}(\mathrm{Gr})-\mathrm{COOH})$ группами работа выхода больше, чем у заполненной водородом кромки. Наша модель относится к иной системе - полубесконечному графену с одной кромкой. С качественной точки зрения результаты работы [1] можно понять, опираясь на теорию адсорбции $[11,16]$.

Определим для начала энергии адчастиц $\varepsilon_{a}=$ $=\phi_{\mathrm{Gr}}-(I+A) / 2$. Воспользовавшись данными справочника [19], для Н, О и ОН получим $\varepsilon_{a}(\mathrm{H})=-2.68$ эВ, $\varepsilon_{a}(\mathrm{O})=-3.04$ эВ, $\varepsilon_{a}(\mathrm{OH})=-2.07$ эВ. В соответствии c теорией адсорбции для чисел заполнения „адсорбированных“ на кромке графена изолированных частиц имеем неравенство $n_{a}(\mathrm{O})>n_{a}(\mathrm{H})>n_{a}(\mathrm{OH})>1$. То же неравенство справедливо для модулей соответствующих зарядов: $Z_{a}=1-n_{a}<0$. Так как вызванное адсорбцией изменение работы выхода графена $\Delta \phi_{\mathrm{Gr}} \propto-Z_{a}[11,16]$, получаем увеличения работы $\Delta \phi_{\mathrm{Gr}}(\mathrm{O})>\Delta \phi_{\mathrm{Gr}}(\mathrm{H})>\Delta \phi_{\mathrm{Gr}}(\mathrm{OH})$, т. е. результат работы [1]. На наш взгляд, однако, игнорирование подложки в подобного рода расчетах существенно снижает ценность полученных результатов. Более того, в неоднородных системах встает вопрос о локальности работы выхода, являющейся, строго говоря, макроскопической характеристикой [24].

В работе [2] рассматривалась энергетика декорирования зигзагообразных кромок азотными комплексами и металлическими атомами. Мы здесь такие вопросы не рассматривали. В [3] показано, что даже слабо связанная с зигзагообразной кромкой треугольного островка графена примесь оказывает сильное влияние на оптические свойства. Расчеты для конкретных случаев отсутствуют.

Подводя итоги, отметим следующее. Во-первых, расчеты показывают, что в случае, когда уровень $\varepsilon_{a}$ лежит вблизи точки Дирака, доминирующую роль играют две узкие энергетические зоны $\omega_{2}(k)$ и $\omega_{3}(k)$. На этом основании можно построить двухзонную модель декорирования. Здесь имеется известная аналогия с теорией электронных свойств графена, где учитываются $\pi$-зоны, 
а $\sigma$-зоны игнорируются. Далее, при $\varepsilon_{a}^{2} \gg \tilde{t}^{2}$ из (7) получим $\omega_{2}(\pi / 2 a) \approx \varepsilon_{a}$ и $\omega_{3}(\pi / 2 a) \approx-2 \tilde{t}^{2} / \varepsilon_{a}$ (см. рис. 2 для случая $\varepsilon_{a}=t$ и $\left.\tau=0.5\right)$, что позволяет сформулировать однозонную модель. Подчеркнем, что в этом случае температурная зависимость чисел заполнения будет определяться именно зоной $\omega_{3}(k)$.

Второй результат расчетов состоит в наличии волны зарядовой плотности вдоль цепочки адатомов углерода типа $(-20),(-10),(00),(10),(20)$, что не удивительно, так как адатомы типа $(-10)$ и (10) взаимодействуют с адчастицами, а адатомы (-20), (00) и (02) с адатомами графена (рис. 1). Равенство $n_{1}=n_{-1}$ является, по-видимому, следствием даже не упрощенной структурной модели, а схемы расчета: вычисление значений $\nu$-множителей в полюсах соответствующих функций Грина дает $C_{1}=C_{-1}$.

И наконец, третий результат - особая роль адатомов типа $(-20),(00)$ и (02), накапливающих самое наибольшее количество электронов при $\varepsilon_{a}>0$ (или дырок при $\left.\varepsilon_{a}<0\right)$ и определяющих переход заряда между адсорбированным комплексом и металлической подложкой.

В настоящей работе мы рассмотрели простейший субстрат - металл. При этом энергетические щели и расходимости плотности состояний свободной системы исчезают. В случае полупроводниковой подложки из-за наличия запрещенной зоны все обстоит намного сложнее: возможно существование узких полос сплошного спектра в области запрещенной зоны субстрата (см., например, [25]). Такая электронная структура увеличивает в принципе возможности управления свойствами графеновых островков.

\section{Список литературы}

[1] R. Taira, A. Yamanaka, S. Okada. Appl. Phys. Express, 9, 115102 (2016).

[2] M.R. Mananghaya, G.N. Santos, D. Yu, C. Stampfl. Sci. Rep. 7, 15727 (2017).

[3] Z.S. Sadeq, R.A. Muniz, J.E. Sipe. Phys. Rev. Mater., 2, 014001 (2018)

[4] K. Nakada, M. Fujita, G. Dresselhaus, M. Dresselhaus. Phys. Rev. B, 54, 17954 (1996).

[5] Y. Miyamoto, K. Nakada, M. Fujita. Phys. Rev. B, 59, 9858 (1999).

[6] S. Okada, M. Igami, K. Nakada, A. Oshiyama. Phys. Rev. B, 62, 9896 (2000).

[7] M.P. Levendorf, C.-J. Kim, L. Brown, P.Y. Huang, R.W. Havener, D.A. Muller, J. Park. Nature, 488, 627 (2012).

[8] Z. Liu, L. Ma, G. Shi, W. Zhou, Y. Gong, S. Lei, X. Yang, J. Zhang, J. Yu, K.P. Hackenberg, A. Babakhani, J.-C. Idrobo, R. Vajtai, J. Lou, P.M. Ajayan. Nature Nanotechnology, 8, 119 (2013).

[9] G.C. Loh, R. Pandey. J. Mater. Chem. C, 3, 5918 (2015).

[10] С.Ю. Давыдов. ФТТ, 60, 1389 (2018).

[11] С.Ю. Давыдов. Теория адсорбции: метод модельных гамильтонианов (СПб., изд-во СПбГЭТУ ЛЭТИ, 2013). twirpx.com/file/1596114/ .
[12] С.Ю. Давыдов. ФТТ, 59, 1650 (2017).

[13] J. Cserti. Am. J. Phys., 68, 896 (2000).

[14] G. Jose, R. Malla, V. Srinivasan, A. Sharma, S. Gangadharaiah. arXiv: $1711.08204 \mathrm{v} .1$.

[15] D.J. Klein. Chem. Phys. Lett., 217, 261 (1994).

[16] С.Ю. Давыдов, А.А. Лебедев, О.В. Посредник. Элементарное введение в теорию наносистем (СПб., Лань, 2014).

[17] С.В. Вонсовский, Ю.А. Изюмов, Э.3. Курмаев. Сверхпроводимость переходных металлов, их сплавов $u$ соединений (М., Наука, 1977) гл. V.

[18] С.Ю. Давыдов. ФТТ, 60, 1815 (2018).

[19] Физические величины. Справочник, под ред. И.С. Григорьева, Е.3. Мейлихова. (М., Энергоатомиздат, 1991).

[20] D. Niesner, N. Fauster. J. Phys.: Condens. Matter, 28, 393001 (2014).

[21] T.L. Einstein. Crit. Rev. Sol. St. Mater. Sci., 7, 261 (1978).

[22] С.Ю. Давыдов. ФТТ, 60, 808 (2018).

[23] У. Харрисон. Электронная структура и свойства твердых тел (М., Мир, 1983) т. 1.

[24] С.Ю. Давыдов. ЖТФ, 49, 211 (1979).

[25] С.Ю. Давыдов. ФТТ, 58, 1182 (2016).

Редактор Г.А. Оганесян

\section{Chain model for the zigzag edge decoration of graphene}

\section{S.Yu. Davydov}

loffe Institute,

194021 St. Petersburg, Russia

Abstract On the basis of the brick-type lattice, topologically equivalent to the graphene lattice, simple structural model for the decorated zigzag edge of graphene is constructed. The analytical expressions for the energy bands, densities of states and occupation numbers are found both for the free system and system formatted on metallic substrate. 